\title{
Comprehensive Sequential Successful Therapy Comprising Chemical Peeling, Iontophoresis and Topical Vitamin C for Postinflammatory Hyperpigmentation in Acne Vulgaris
}

\author{
Ichiro Kurokawa \\ Department of Dermatology, Meiwa Hospital, Nishinomiya, Japan \\ Email: kurokawa.i@meiwa-hospital.com
}

How to cite this paper: Kurokawa, I. (2020) Comprehensive Sequential Successful Therapy Comprising Chemical Peeling, Iontophoresis and Topical Vitamin C for Postinflammatory Hyperpigmentation in Acne Vulgaris. Journal of Cosmetics, Dermatological Sciences and Applications, 10, 99-103.

https://doi.org/10.4236/jcdsa.2020.103010

Received: June 18, 2020

Accepted: July 10, 2020

Published: July 13, 2020

Copyright $\odot 2020$ by author(s) and Scientific Research Publishing Inc. This work is licensed under the Creative Commons Attribution International License (CC BY 4.0).

http://creativecommons.org/licenses/by/4.0/

\begin{abstract}
A 20-year-old man presented with for 6-month history of facial acne. He had erythema and red papules on the face secondary to BPO-induced contact dermatitis. He was administered topical corticosteroid. Contact dermatitis improved with this treatment, and he had red papules, comedones, prominent postinflammatory hyperpigmentation (PIH), postinflammatory erythema (PIE), erosions and erythema associated with acne vulgaris. He was subsequently treated with oral minocycline $100 \mathrm{mg} / \mathrm{d}$ and topical adapalene and ozenoxacin lotion once daily for 3 months. The inflammatory lesions and comedo subsided; however, PIH, PIE, atrophic scar and erosion persisted. During 3 months, the patient underwent chemical peeling using 20\% glycolic acid (GA) and subsequent vitamin $C$ iontophoresis twice at 1-month intervals. He showed almost disappearance of red papules and comedones but persistent PIH, PIE and erosion after 3 months of treatment. He was thereafter prescribed topical glyceryl-octyl-ascorbic acid/ascorbyl 2-phosphate 6-palmitate/DL-a-tocopherol phosphate complex for local application twice daily for 3 months. After 7 months of treatment, PIH, PIE, erosion and atrophic scar faded significantly with only trace residual erosions, atrophic scar and PIH. Subsequently, he was prescribed local application of $2 \%$ isostearyl-L-ascorbic acid gel vitamin $\mathrm{C}$ gel twice daily for 3 months. After 15 months, PIH, PIE, erosion and atrophic scar disappeared completely with significant improvement. Comprehensive sequential therapy resulted in significant improvement. It is suggested that medical treatment using systemic and topical antimicrobials and topical adapalene reduces inflammatory lesions and comedones initially. Subsequent chemical peeling using GA and vitamin $\mathrm{C}$ iontophoresis could improve $\mathrm{PIH}$. These synergistic effects might
\end{abstract}


have contributed to the significant improvement observed in this case. Comprehensive sequential treatment using chemical peeling, vitamin $\mathrm{C}$ iontophoresis and topical vitamin $\mathrm{C}$ can be a useful treatment strategy for $\mathrm{PIH}$ in acne vulgaris.

\section{Keywords}

Therapy, Facial Acne, Postinflammatory Hyperpigmentation

A 20-year-old man presented with for 6-month history of facial acne. He was treated with topical benzoyl peroxide (BPO). Two weeks prior to presentation, he developed itchy erythema and red papules on the face secondary to BPO-induced contact dermatitis. At the first visit, BPO was discontinued, and he was administered topical corticosteroid (hydrocortisone butyrate). Contact dermatitis improved with this treatment, and he had red papules, comedones, prominent postinflammatory hyperpigmentation $(\mathrm{PIH})$, postinflammatory erythema (PIE), erosions and erythema associated with acne vulgaris (Figure 1(a)) He was subsequently treated with oral minocycline $100 \mathrm{mg} / \mathrm{d}$ and topical adapalene and ozenoxacin lotion once daily for 3 months. The inflammatory lesions and comedo subsided; however, PIH, PIE, atrophic scar and erosion persisted. During 3 months, the patient underwent chemical peeling using $20 \%$ glycolic acid (GA) and subsequent vitamin $C$ iontophoresis twice at 1-month intervals. He showed almost disappearance of red papules and comedones but persistent PIH, PIE and erosion after 3 months of treatment (Figure 1(b)).

He was thereafter prescribed topical glyceryl-octyl-ascorbic acid/ascorbyl 2-phosphate 6-palmitate/DL-a-tocopherol phosphate complex for local application twice daily for 3 months. After 7 months of treatment, PIH, PIE, erosion and atrophic scar faded significantly with only trace residual erosions, atrophic scar and PIH (Figure 1(c)). Subsequently, he was prescribed local application of $2 \%$ isostearyl-L-ascorbic acid gel vitamin $\mathrm{C}$ gel twice daily for 3 months. After 15 months, PIH, PIE, erosion and atrophic scar disappeared completely with significant improvement (Figure 1(d)).

Guidelines for the treatment of acne vulgaris 2017 were published in Japan [1]. However, these guidelines do not describe treatment for PIH in patients with acne vulgaris [1]. Persistent PIH is more psychologically disturbing more than the original or residual acne itself and negatively affects patients' quality of life [2]. PIH corresponds to the area affected by inflammation [2]. Chemical peeling and subsequent iontophoresis are non-surgical treatments recommended for $\mathrm{PIH}$. Reportedly, topical amphiphilic vitamin $\mathrm{C}$ derivatives that can penetrate into follicles are among the available treatments prescribed for PIH in patients with acne [3]. Comprehensive sequential therapy resulted in significant improvement. It is suggested that medical treatment using systemic and topical antimicrobials and topical adapalene reduces inflammatory lesions and comedones 


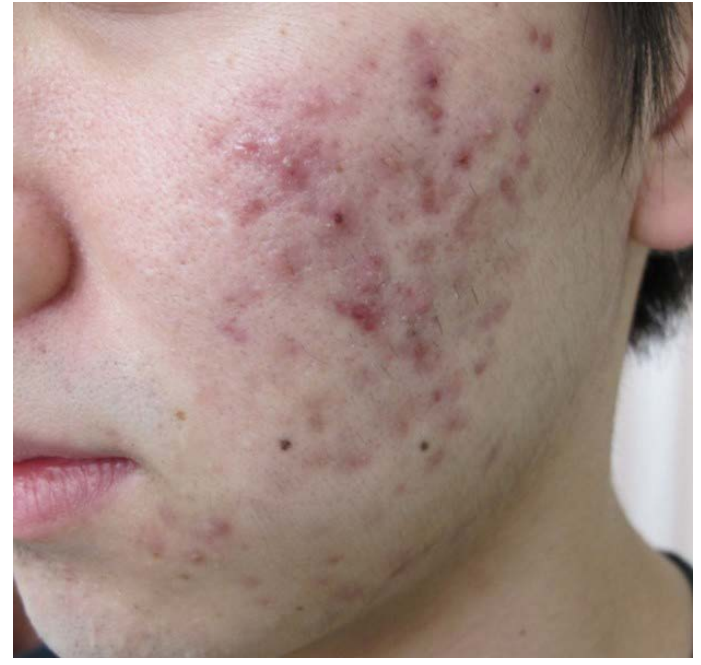

(a)

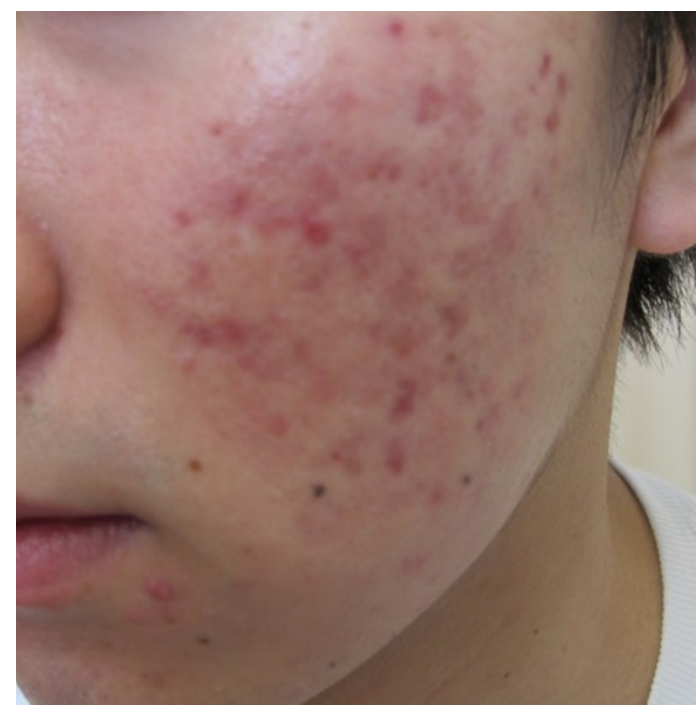

(b)

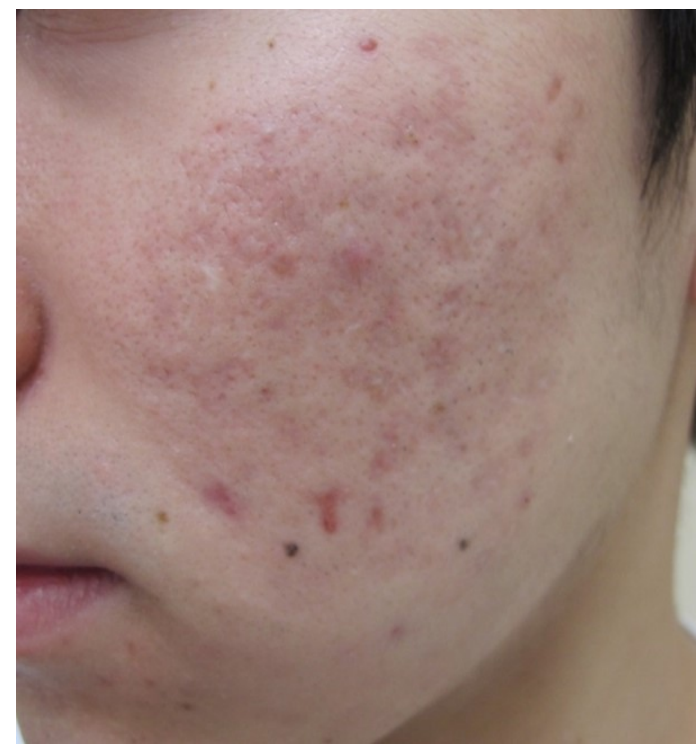

(c) 


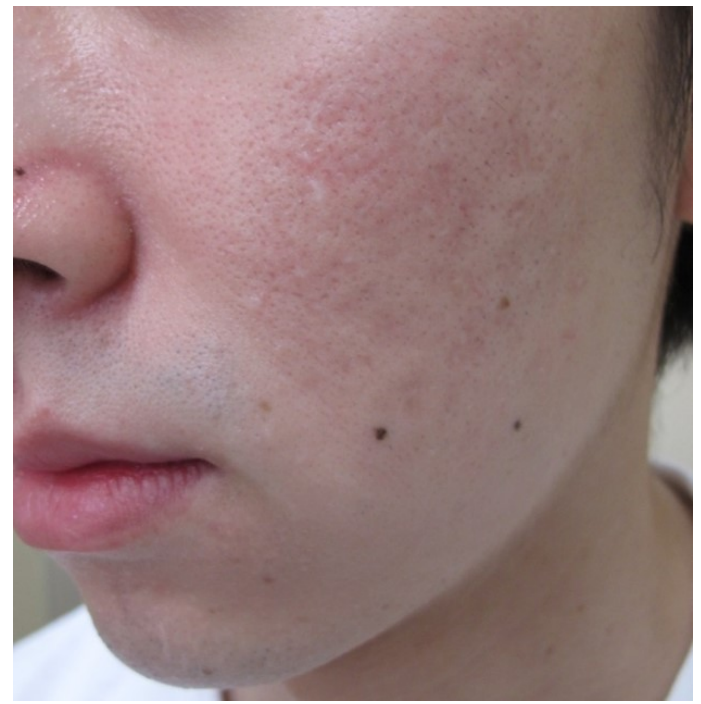

(d)

Figure 1. (a) Clinical manifestation before the treatment: red papules, comedo, prominent PIH, PIE, and erosion were observed. (b) Clinical manifestation 3 months after the treatment: red papules and comedones almost disappeared, but persistent PIH, PIE and erosion were observed. (c) Clinical manifestation 7 months after the treatment: PIH, PIE, erosion and atrophic scar faded significantly with only trace residual erosions, atrophic scar and PIH. (d) Clinical manifestation 15 months after the treatment: PIH, PIE, erosion and atrophic scar disappeared completely with significant improvement.

initially. Subsequent chemical peeling using GA and vitamin C iontophoresis could improve PIH by promoting self-renewal of epidermal basal cells, collagen synthesis by fibroblasts and de-roofing as well as inhibition of melanogenesis. Vitamin C iontophoresis produces anti-inflammatory effects and inhibits IL-1b and TNF-a, inflammatory melanin in wounds and tyrosinase and reactive oxygen.

These synergistic effects might have contributed to the significant improvement observed in this case. PIH is a challenging and distressing sequela in patients with acne [4]. PIH typically will fade in 3 to 18 months [5]. Further accumulation of cases is necessary to evaluate the clinical efficacy to confirm the role of the comprehensive sequential therapy outlined in this report.

Comprehensive sequential treatment using chemical peeling, vitamin $\mathrm{C}$ iontophoresis and topical vitamin $\mathrm{C}$ can be a useful treatment strategy for PIH in acne vulgaris.

\section{Conflicts of Interest}

The author declares no conflicts of interest regarding the publication of this paper.

\section{References}

[1] Hayashi, N., Akamatsu, H., Iwatsuki, K., et al. (2018) Japanese Dermatological Association Guidelines: Guidelines for the Treatment of Acne Vulgaris 2017. Journal 
of Dermatology, 45, 898-935. https://doi.org/10.1111/1346-8138.14355

[2] Callendar, V.D. (2004) Acne in Ethnic Skin: Special Considerations for Therapy. Dermatologic Therapy, 17, 184-195.

https://doi.org/10.1111/j.1396-0296.2004.04019.x

[3] Kurokawa, I., Yoshioka, M. and Ito, S. (2019) Split-Face Comparative Clinical Trial Using Glyceryl-Octyl-Ascorbic Acid/Ascorbyl 2-Phosphate 6-Palmitate/DL-a-tocopherol Phosphate Complex Treatment for Postinflammatory Hyperpigmentation, Postinflammatory Erythema and Atrophic Scar in Acne Vulgaris. Journal of Dermatology, 46, e347-e348. https://doi.org/10.1111/1346-8138.14930

[4] Goh, C.L., Abad-Casintahan, F., Chow, S.K., et al. (2014) Evaluating Acne-Related Post-Inflammatory Hyperpigmentation Is a Challenge Even amongst Experts. Journal of Dermatology, 41, 1106-1108. https://doi.org/10.1111/1346-8138.12667

[5] Goodman, G.J. (2001) Post-Acne Scarring: A Short Review of Its Pathophysiology. Australasian Journal of Dermatology, 42, 84-90.

https://doi.org/10.1046/j.1440-0960.2001.00487.x 